\title{
A quantitative MRI investigation of the association between iliotibial band syndrome and patellofemoral malalignment
}

\author{
Jia Li", Bo Sheng", Lanyu Qiu, Fan Yu, Fa-Jin Lv, Fu-Rong Lv, Haitao Yang \\ Department of Radiology, the First Affiliated Hospital of Chongqing Medical University, Chongqing, China \\ \#These authors contributed equally to this work.
}

Correspondence to: Haitao Yang. Department of Radiology, the First Affiliated Hospital of Chongqing Medical University, 1 Youyi Road, Yuzhong District, Chongqing 400016, China. Email : frankyang119@126.com.

\begin{abstract}
Background: The iliotibial band (ITB) has a wide patellar insertion that provides lateral restraint to the patella and maintains the patellofemoral joint's stability. There has been limited investigation into the relationship between patellofemoral malalignment and iliotibial band syndrome (ITBS).

Methods: We retrospectively analyzed 47 knees with ITBS by retrieving magnetic resonance imaging (MRI) data collected over an approximately 6-year period from our database. The Insall-Salvati ratio, lateral patellofemoral angle (LPA), lateral patellar tilt (LPT), lateral trochlear length (LTL), angle of the nonweight-bearing facet of the lateral femoral condyle (nwb-LFCA), and the ITB-lateral femoral condyle (ITLFC) distance were measured on MR images. The knees of 47 age- and gender-matched subjects were enrolled as the normal group.

Results: In the ITBS group, over one third $(34 \%, 16 / 47)$ of knees had abnormal patellofemoral measurements, including $8(17 \%, 8 / 47)$ knees with patellar alta, $11(23.4 \%, 11 / 47)$ knees with an abnormally decreased LPA, and $5(10.6 \%, 5 / 47)$ knees with an abnormally increased LPT indicating lateral patellar tilt. Moreover, 8 knees had simultaneous combinations of two or three abnormality parameters, and 8 (17\%, 8/47) knees presented with superolateral Hoffa's fat pad edema. The Insall-Salvati ratio, LPT, and nwbLFCA in the ITBS group were significantly higher than those in the normal group $(\mathrm{P}=0.001, \mathrm{P}<0.001$, and $\mathrm{P}<0.001$, respectively); the LPA and IT-LFC distances in the ITBS group were significantly lower $(\mathrm{P}=0.003$, $\mathrm{P}<0.001$, respectively) than those in the normal group. There were mild to moderate correlations between the MRI parameters and ITBS $(\mathrm{P}=0.006, \mathrm{P}<0.001$, respectively).

Conclusions: This study confirmed that a higher position or lateral tilt of the patella and a steeper morphology of the anterior part of the lateral femoral condyle were associated with the development of ITBS, which is helpful in understanding and further exploring the mechanism of ITBS.
\end{abstract}

Keywords: Iliotibial band syndrome (ITBS); patella; lateral femoral condyle; magnetic resonance imaging

Submitted Sep 27, 2020. Accepted for publication Mar 10, 2021.

doi: 10.21037/qims-20-1101

View this article at: http://dx.doi.org/10.21037/qims-20-1101

\section{Introduction}

The iliotibial band (ITB) is a longitudinal thick fibrous connective tissue extending along the thigh's lateral part that acts to coordinate muscle function and stabilize the knee during flexion and extension. Iliotibial band syndrome (ITBS) is an overuse injury well recognized as a common cause of lateral knee pain and associated with various activities, particularly running and cycling (1-3). In runners, the incidence of ITBS is reported to be as high as $7-14 \%(1-3)$. Causes of ITBS include biomechanical abnormalities (2), excessive friction of the ITB over the lateral femoral epicondyle during repetitive knee movement, and compression of the fat and connective 

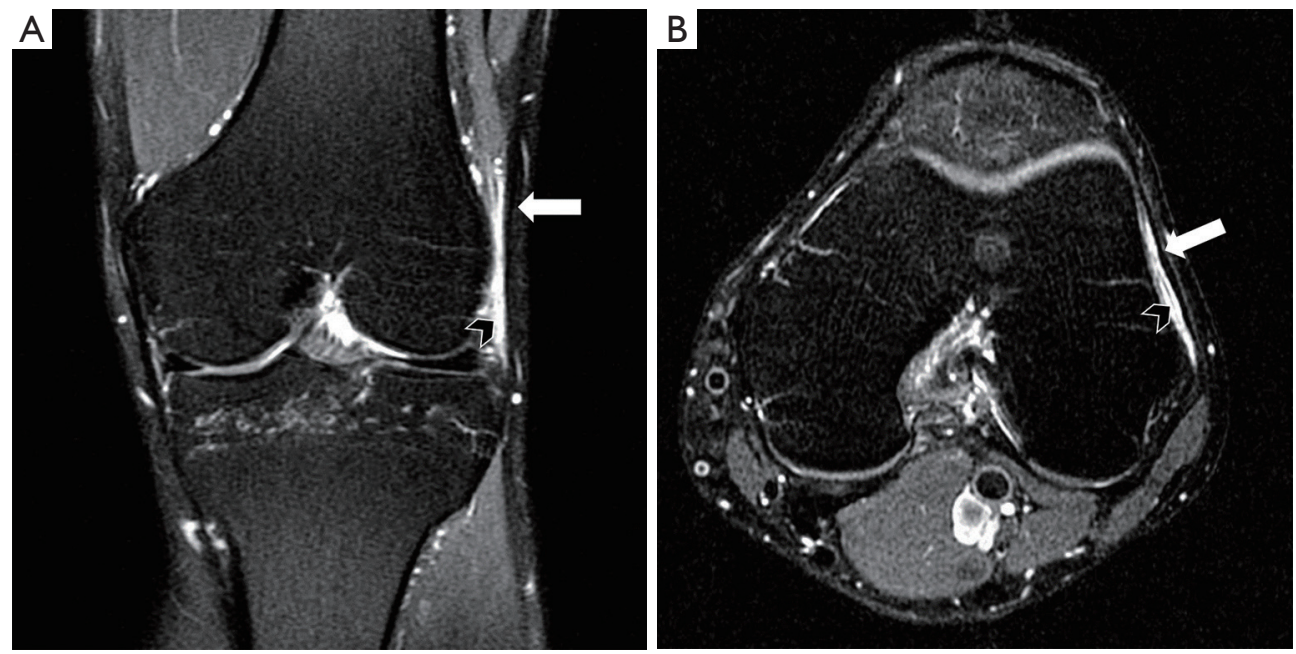

Figure 1 Magnetic resonance imaging (MRI) findings in patients with ITBS. MR images of a 30-year-old man with lateral pain in both knees for 3 months, aggravated after running. The patient persisted in running five kilometers twice a week for 2 years. The coronal (A) and axial (B) intermediate-weighted MR images with fat saturation of the left knee show focal hyperintense edema-like signals (black arrowhead) within the fat space between the ITB (white arrow) and the lateral femoral condyle. ITBS, iliotibial band syndrome; ITB, iliotibial band.

tissue between the ITB and the lateral femoral epicondyle, all of which produce pain and inflammation (4). In ITBS patients, there is classic pain or tightness $2-3 \mathrm{~cm}$ above the lateral joint line (2), and lateral knee pain is aggravated by repetitive knee flexion, particularly at $20^{\circ}-30^{\circ}$ of knee flexion (5).

The etiology of ITBS involves both intrinsic and extrinsic factors $(6,7)$, and the risk factors for ITBS include past history, static and dynamic alignment in the lower extremities, and gender (8). Previous studies have identified that excessive motion of the hip and knee in the frontal and transverse planes are important factors in the development of ITBS $(6,9,10)$. Some studies have focused on the relationship between dynamic alignment in the lower extremities and ITBS. Shen et al. (11) found that ITBS might be related to the lack of timely gait adjustment, excessive trunk inclination, and anterior pelvic tilt angle. Taunton et al. (1) reported that $33-55 \%$ of runners with genu varum knee alignments had experienced ITBS. Everhart et al. (12) reported that a greater lateral epicondyle prominence was significantly associated with ITBS.

At least five distal insertions of the ITB, including the proximal tibia, distal femur, patella, and joint capsule, have been described (13-15). Except for the direct insertion of the ITB on Gerdy's tubercle, the ITB insertions on the distal femur include the linea aspera and the lateral femoral epicondyle (13-15). Also, the ITB has a wide patellar insertion that is fused into the lateral patellar retinaculum, and this provides lateral restraint to the patella and maintains the stability of the patellofemoral joint $(13,15)$. A previous in vitro specimen study reported ITB tightness reduced lateral patellar stability (16). However, there have been few studies focused on the morphological characteristics of the patellar insertion of the ITB and the correlation between subtle patellofemoral instability and ITBS. In this study, we present our contribution to the exploration of the association between patellofemoral malalignment and ITBS by comparing the difference in patellar position and morphology of the anterior part of the lateral femoral condyle between an ITBS group and normal group using in vivo magnetic resonance imaging (MRI) quantitative measurements.

\section{Methods}

\section{Participants}

The Ethics Committee for Human Research of our institution approved this study, which was conducted following the Helsinki Declaration. We retrospectively reviewed patients with ITBS who underwent MRI from January 2014 to October 2019 using picture archiving and communication systems from our department. Inclusion criteria were as follows: (I) patients with typical clinical presentations and physical examinations consistent with ITBS; (II) an edema-like signal intensity located between the ITB and the lateral femoral condyle on MRI $(15,17,18)$ (Figure 1). Exclusion criteria were as follows: (I) patients 


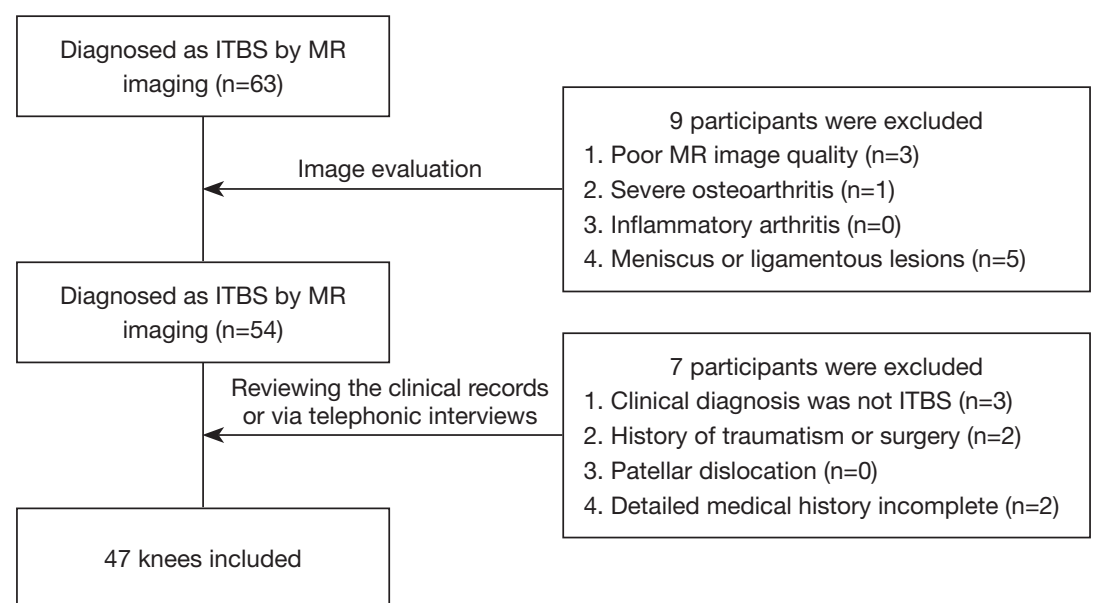

Figure 2 Flowchart for selection of participants in the ITBS group. ITBS, iliotibial band syndrome.

with a history of trauma, arthroscopy, or surgery of the knee; (II) presence of patellar dislocation, meniscus, or ligamentous lesions; (III) presence of severe osteoarthritis ( $\geq$ KL grade 3) or inflammatory arthritis; (IV) a length discrepancy in the lower limbs, or a history of genu varus or genu valgus (Figure 2). All clinical information was acquired by reviewing the clinical records or, if the clinical records were unclear, a medical history was conducted by telephone. An age- and gender-matched case-control study design was used. Participants in the control group were selected from subjects who showed normal knee MRI manifestations without any detectable alignment abnormalities or structural lesions on MRI during the same period at our department.

\section{MRI protocol}

MRI was performed using 1.5-T MRI equipment (Magnetom Essenza; Siemens Healthcare) with an extremity matrix knee coil (a Tim coil). The imaging protocol was as follows: sagittal and coronal T1 turbo spin echo (repetition time/echo time $306 / 12 \mathrm{~ms}$, the field of view $16 \mathrm{~cm}$, matrix size $320 \times 320$, slice thickness $4 \mathrm{~mm}$ ), sagittal T2 turbo spin echo (repetition time/echo time $3,220 / 99 \mathrm{~ms}$, the field of view $16 \mathrm{~cm}$, matrix size $320 \times 320$, slice thickness $4 \mathrm{~mm}$ ), sagittal and coronal intermediate-weighted turbo spin echo with fat saturation (repetition time/echo time 2,800/38 ms, the field of view $16 \mathrm{~cm}$, matrix size $256 \times 256$, slice thickness $4 \mathrm{~mm}$ ), and axial intermediate-weighted turbo spin echo with fat saturation (repetition time/echo time 2,800/51 ms, the field of view $16 \mathrm{~cm}$, matrix size $232 \times 256$, slice thickness $4 \mathrm{~mm}$ ).

\section{Image analyses}

All MRI images were independently reviewed by two musculoskeletal radiologists (Qiu and Sheng, with 3 and 12 years of clinical experience, respectively), and the presence of edema was determined as a focal high signal intensity in the space between the ITB and the lateral femoral condyle on intermediate-weighted turbo spin echo with fat saturation MR images $(15,17,18)$. It needed to be observed on at least two consecutive slices and simultaneously displayed in the axial and coronal positions. The MRI measurements were performed by two other fellowship-trained musculoskeletal radiologists ( $\mathrm{Li}$ and Yu, with 6 and 17 years of clinical experience, respectively) after all the two groups' data were mixed and blindly and randomly distributed. All measurements were repeated by one of the radiologists $(\mathrm{Li})$, and the interval between the first and second reading was at last 4 weeks.

Three quantitative parameters for evaluating patellar position were assessed on the images of each MRI examination, including the Insall-Salvati ratio, the lateral patellofemoral angle (LPA), and the lateral patellar tilt (LPT). The Insall-Salvati ratio (19) was calculated as the patellar tendon length divided by the maximum patellar length on sagittal MRI (Figure $3 A$ ). The Insall-Salvati ratio was used to reflect the patella's height, and the threshold was defined as $>1.2$. The LPA and LPT were used to evaluate patellar tilt's degree and were measured at axial slices where the patella was the widest on an intermediateweighted fast spin-echo MRI $(20,21)$ (Figure 3B,C). The LPA was the angle between the lateral patellar facet and the femoral condyles' anterior aspect, where an LPA $>8^{\circ}$ was 

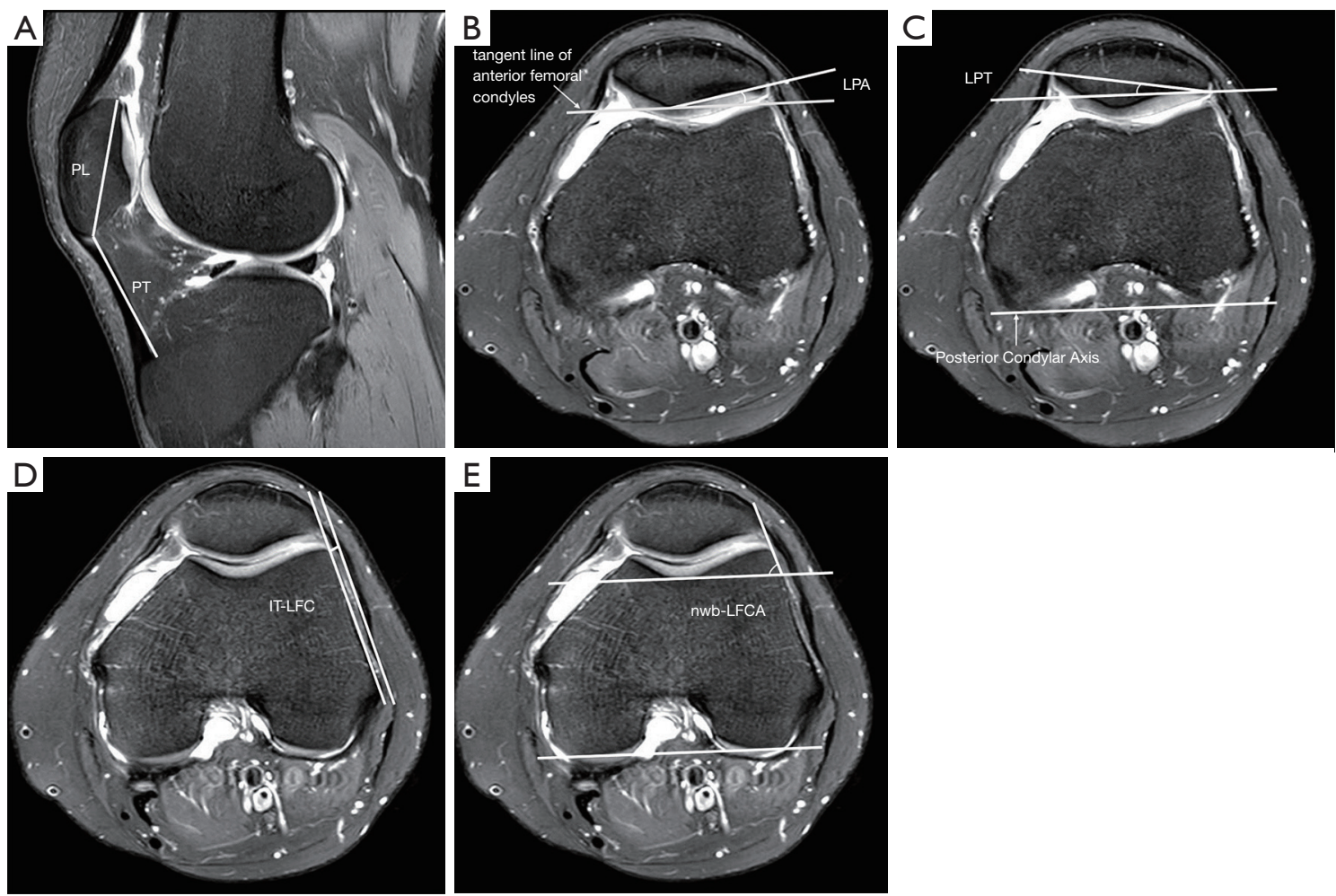

Figure 3 Methods for determining the Insall-Salvati ratio, IT-LFC distance, nwb-LFCA, LPA, and LPT using manual measurements on MRI. Demonstration of measurements of the related parameters on MR images. (A) The Insall-Salvati ratio is measured on the sagittal intermediate-weighted MRI and calculated as the patellar tendon length/maximum patellar length. (B) The lateral patellofemoral angle (LPA) is the angle between the lateral patellar facet and the tangent line of the anterior femoral condyles. (C) The lateral patellar tilt (LPT) is defined as the angle between the patellar axis (the line between the medial and lateral articular margins of the patella) and the tangent line of the posterior femoral condyles. (D) With the non-weight-bearing facet of the lateral femoral condyle as the reference line, the vertical distance from the reference line at the intersection of the non-weight-bearing facet of the lateral femoral condyle and the lateral surface of the trochlea to the medial margin of the ITB was defined as the ITB-lateral femoral condyle (IT-LFC) distance. (E) The angle of the nonweight-bearing facet of the lateral femoral condyle (nwb-LFCA) was the angle between the non-weight-bearing facet of the anterior part of lateral femoral condyle and the tangent line of the posterior femoral condyles. (B,C) were measured at the slice of the lower edge of the patella; (D,E) were measured at axial slices where the lateral femoral epicondyle was the most prominent.

defined as normal (20). The LPT was the angle between the patellar axis (between the medial and lateral articular margins of the patella) and the tangent line of the posterior femoral condyles, where an LPT $>12^{\circ}$ was considered abnormal (21).

Also, this study assessed the morphologic features of the ITB-lateral femoral condyle (IT-LFC) distance (Figure 3D) and the lateral femoral condyle, including the angle of the non-weight-bearing facet of the lateral femoral condyle (nwbLFCA) (Figure 3E). They were measured at axial slices where the lateral femoral epicondyle was the most prominent on an intermediate-weighted turbo spin-echo MRI. The nwb-LCFA was the angle between the non-weight-bearing facet of the anterior part of the lateral femoral condyle and the posterior femoral condyle's tangent line. With the non-weight-bearing facet of the lateral femoral condyle as the reference line, the vertical distance from the reference line at the intersection of the non-weight-bearing facet of the lateral femoral condyle and the lateral surface of the trochlea to the medial margin of the ITB was defined as the IT-LFC distance.

\section{Statistical tests}

In this study, descriptive data were extracted from the 
Table 1 Descriptive data

\begin{tabular}{lcc}
\hline Characteristic & $\begin{array}{c}\text { ITBS group } \\
(\mathrm{n}=47)\end{array}$ & $\begin{array}{c}\text { Normal group } \\
(\mathrm{n}=47)\end{array}$ \\
\hline Age, years & $35 \pm 10$ & $35 \pm 10$ \\
Gender, $\mathrm{n}(\%)$ & $25(53.2)$ & $25(53.2)$ \\
Male & $22(46.8)$ & $22(46.8)$ \\
Female & & \\
Side, $\mathrm{n}(\%)$ & $27(57.4)$ & $26(55.3)$ \\
Right & $20(42.6)$ & $21(44.7)$ \\
Left & $8(17.0)$ & - \\
Patellar alta, $\mathrm{n}(\%)$ & $11(23.4)$ & - \\
LPA $<8^{\circ}, \mathrm{n}(\%)$ & $5(10.6)$ & - \\
LPT $>12^{\circ}, \mathrm{n}(\%)$ & $8(17.0)$ & - \\
$\begin{array}{l}\text { With Superolateral Hoffa fat } \\
\text { pad edema, } \mathrm{n}(\%)\end{array}$ & & - \\
\hline
\end{tabular}

LPA, lateral patellofemoral angle; LPT, lateral patellar tilt; ITBS, iliotibial band syndrome.

demographic data. The subjects were categorized into the ITBS group and the control group according to the inclusion and exclusion criteria. Intraclass correlation coefficients (ICCs) were used to assess the intra- and inter-reader reliability of the MRI quantitative measures. Continuous variables with a normal distribution were expressed as the mean \pm standard deviation, and the Student's $t$-test was used to detect differences. Continuous variables with abnormal distribution were expressed as the median (quartile) and analyzed using the Wilcoxon test for all values of the two groups' quantitative MRI patellofemoral parameters. The Spearman correlation was used to analyze the relationship between all of the parameters above and ITBS. All statistical analyses were performed using Windows software SPSS (version 17.0; SPSS, Chicago, IL, USA), and the significance level was set at 0.05 .

\section{Results}

\section{Demographic characteristics of the subjects in the two groups}

During the study period, a total of 47 knees $(27$ left, 20 right) of 47 patients in the ITBS group (25 male and 22 female, age range $16-62$ years, mean age $35 \pm 10$ years) were included. There were 47 knees (26 left, 21 right) of age- and gender-matched participants in the normal group
(25 male and 22 female, age range 16-62 years, mean age $35 \pm 10$ years) (Table 1$)$.

\section{Intra- and inter-observer reliability of quantitative MRI measurements}

The intra-reader ICCs for the Insall-Salvati ratio, ITLFC distance, nwb-LFCA, LPA, and LPT quantitative measurements ranged from 0.872 to 0.947 , and the interreader ICCs ranged from 0.868 to 0.931 , as shown in Table 2 , indicating good intra- and inter-reader reliability.

\section{Incidence of abnormal patellofemoral measurements in ITBS}

In the ITBS group, there were $8(17 \%, 8 / 47)$ knees with an increased Insall-Salvati ratio indicating patellar alta, $11(23.4 \%, 11 / 47)$ knees with an LPA less than $8^{\circ}$, and 5 $(10.6 \%, 5 / 47)$ knees with an LPT more than $12^{\circ}$ indicating lateral patellar tilt. Of these, 8 knees simultaneously had a combination of two or three abnormality parameters, so in total, $16(34 \%, 16 / 47)$ knees had abnormal patellofemoral measurements. Also, $8(17 \%, 8 / 47)$ knees in the ITBS group presented with superolateral Hoffa's fat pad edema (Table 1).

\section{Comparison between the ITBS group and the normal group}

All five measurement parameters showed significant differences between the ITBS group and the normal group. The Insall-Salvati ratio, LPT, and nwb-LFCA in the ITBS group were significantly higher than those of the normal group $(\mathrm{P}=0.001, \mathrm{P}<0.001$, and $\mathrm{P}<0.001$, respectively), and the LPA and IT-LFC distance were significantly lower $(\mathrm{P}=0.003, \mathrm{P}<0.001$, respectively) in the ITBS group than in the normal group (Table 3).

\section{Correlation between MRI measurements and ITBS}

All five measurement parameters were correlated with ITBS. The Nwb-LFCA showed a moderate positive correlation with ITBS $(\mathrm{P}<0.001)$, and the IT-LFC distance showed a moderate negative correlation with ITBS $(\mathrm{P}<0.001)$. The Insall-Salvati ratio and LPT showed a mild positive correlation with ITBS $(\mathrm{P}<0.001)$, and the LPA showed a mild negative correlation with ITBS $(\mathrm{P}=0.006)$. The specific results are shown in Table 4. 
Table 2 The intra- and inter-observer agreement between two radiologists of the quantitative MRI measurements

\begin{tabular}{|c|c|c|c|c|c|c|}
\hline Quantitative parameters & \multicolumn{3}{|c|}{ Intra-observer } & \multicolumn{3}{|c|}{ Inter-observer } \\
\hline Insall-Salvati ratio & $0.927(0.846-0.965)$ & 13.653 & $<0.001$ & $0.931(0.855-0.967)$ & 14.469 & $<0.001$ \\
\hline LPA & $0.947(0.889-0.975)$ & 18.937 & $<0.001$ & $0.879(0.745-0.942)$ & 8.235 & $<0.001$ \\
\hline LPT & $0.929(0.850-0.966)$ & 14.017 & $<0.001$ & $0.886(0.761-0.946)$ & 8.785 & $<0.001$ \\
\hline nwb-LFCA & $0.914(0.819-0.959)$ & 11.625 & $<0.001$ & $0.868(0.723-0.937)$ & 7.586 & $<0.001$ \\
\hline
\end{tabular}

LPA, lateral patellofemoral angle; LPT, lateral patellar tilt; IT-LFC distance, ITB-lateral femoral condyle distance; nwb-LFCA, angle of non-weight-bearing facet of lateral femoral condyle; ICCs, intraclass correlation coefficients.

Table 3 Comparison of MR quantitative parameters between the ITBS and normal groups

\begin{tabular}{|c|c|c|c|c|c|}
\hline Group & Insall-Salvati ratio & LPA & LPT & IT-LFC distance $(\mathrm{mm})$ & nwb-LFCA \\
\hline Normal group & $1.024 \pm 0.064$ & $11.391^{\circ} \pm 2.513^{\circ}$ & $5.950^{\circ} \pm 2.486^{\circ}$ & $2.560 \pm 0.905$ & $62.311^{\circ} \pm 3.821^{\circ}$ \\
\hline $\mathrm{t}$ & -3.492 & 3.044 & -3.859 & 3.876 & -6.035 \\
\hline $\mathrm{P}$ & 0.001 & 0.003 & $<0.001$ & $<0.001$ & $<0.001$ \\
\hline
\end{tabular}

LPA, lateral patellofemoral angle; LPT, lateral patellar tilt; IT-LFC distance, ITB-lateral femoral condyle distance; nwb-LFCA, angle of non-weight-bearing facet of lateral femoral condyle; ITBS, Iliotibial band syndrome.

Table 4 Correlation analysis between measurement parameters and ITBS

\begin{tabular}{|c|c|c|c|c|c|}
\hline Statistical value & Insall-Salvati ratio & LPA & LPT & IT-LFC distance & nwb-LFCA \\
\hline$P$ & $<0.001$ & 0.006 & $<0.001$ & $<0.001$ & $<0.001$ \\
\hline
\end{tabular}

r, correlation coefficient; LPA, lateral patellofemoral angle; LPT, lateral patellar tilt; IT-LFC distance, ITB-lateral femoral condyle distance; nwb-LFCA, angle of non-weight-bearing facet of lateral femoral condyle; ITBS, lliotibial band syndrome.

\section{Discussion}

The main findings of this study were twofold. Firstly, our data showed a relatively high incidence of patellofemoral malalignment in the ITBS patients, with $34 \%$ of cases showing abnormal patellofemoral measurements in patellar height and lateral tilt. These results suggest that a higher position or a more lateral tilt in the patella is associated with a greater risk of developing ITBS. Secondly, ITBS patients presented with a steeper anterior part of the lateral femoral condyle, represented by a larger nwb-LFCA and smaller IT-LFC distance than normal subjects, which may also be a potential risk factor for developing ITBS. These findings support our hypothesis and indicate that an abnormal patella position and abnormal morphology of the anterior part of the lateral femoral condyle may play a role in ITBS.

The patellar height was significantly higher in the ITBS group than in the normal group, and there were 8 (17\%) knees with patellar alta among the ITBS patients. The ITB has a wide patellar insertion and constitutes the lateral patellofemoral ligament complex $(13,15)$ that plays an important role in precluding anterior-posterior movement of the lateral femoral epicondyle (22). A higher patella position allows excessive lateral motion during repetitive knee flexion (23). A more lateral and higher patellar insertion of the ITB might cause the ITB to be relatively loose, thus increasing the ITB rubbing on the lateral epicondyle of the femur. Additionally, a higher patella position may not be able to engage the trochlear groove 
early in knee flexion $(19,24)$, and patella alta is a recognized anatomical factor in patellar instability (24).

The patients with ITBS had an increased lateral tilt of the patella in this study. The LPA in $11(23.4 \%)$ knees and the LPT in 5 (10.6\%) knees exceeded the normal range in the ITBS group. An increased lateral tilt of the patella might result in the ITB being closer to the lateral femoral epicondyle, causing the soft tissues' mechanical impingement between the lateral femoral epicondyle and the ITB. However, in some chronic ITBS cases, the ITB is thickened $(15,25)$, and a thickened ITB might cause the elasticity of the ITB to decrease, which in turn increases the tightness of the ITB, affecting lateral stability of the patella and causing the patella to tilt laterally. This may explain why the LPA and LPT were significantly different between the two groups but still mostly within the normal range. In other words, it is not clear from this retrospective crosssectional study whether ITBS occurred before the changes to the patella or whether the differences in the patella caused the ITBS. We suggest that people with patellar maltracking need to be vigilant about the risk of ITBS.

It is interesting to note that $8(17 \%)$ knees in the ITBS group had superolateral Hoffa's fat pad edema. Superolateral Hoffa's fat pad edema, also known as patellar tendon-lateral femoral condyle friction syndrome, is generally considered to be due to impingement between the patellar tendon and lateral femoral condyle and is also correlated with underlying patellofemoral instability (26-29). Anatomic risk factors for lateral patellar instability include patella alta, increased lateral patellar tilt, and trochlear dysplasia (30). Previous studies have shown that patella alta, lateral displacement and patella tilt, and superolateral Hoffa's fat pad edema are all associated with the occurrence and development of patellofemoral osteoarthritis (31-35). Haj-Mirzaian et al. (33) reported that the optimal cutoff point of the Insall-Salvati ratio as a predictor of lateral patellofemoral osteoarthritis was $\geq 1.14$, which is still within the normal range (0.8-1.2). So, both ITBS and superolateral Hoffa's fat pad edema may be correlated with underlying patellofemoral malalignment but may present different injury patterns.

In addition to the patella position, this study also evaluated the anatomic characteristics of the lateral femoral condyle adjacent to the patellar insertion of the ITB. ITBS patients showed a steeper morphology in the non-weightbearing facet of the lateral femoral condyle than control subjects. In effect, this meant that the larger the nwbLFCA, the closer the lateral femoral condyle and the ITB were. We further measured the distance between the ITB and the non-weight-bearing facet of the lateral femoral condyle, and the results showed that the IT-LFC distance was significantly lower in the ITBS group than in the normal group, which was consistent with the results of the nwb-LFCA. The two parameters could indirectly reflect the reduction in the distance between the lateral femoral epicondyle and the ITB, possibly leading to an increased mechanical impingement between the two structures.

The five quantitative MRI measurement parameters were correlated with ITBS. Although these correlations were mild to moderate, there was a relatively high incidence of abnormal patellofemoral measurements in our data. We believe that patellofemoral malalignment might be associated with ITBS, and the results could provide a whole new perspective for analyzing the risk factors and pathogenesis of ITBS. Many researchers have confirmed the correlation between patellar malalignment or patellar maltracking and patellofemoral osteoarthritis (31-34); therefore, whether patients with ITBS should pay attention to the occurrence of patellofemoral osteoarthritis is also an interesting research direction.

In this study, MR images of knees during an approximately 6-year period were retrieved from our database and analyzed, and there were only 47 knees with ITBS, which is far below the incidence of ITBS reported in the literature. It might be related to the following reasons. Firstly, the diagnosis of ITBS is typically made on clinical presentation and by physical examination $(4,15)$, and usually, only recurrent or refractory cases have an MR scan; therefore, most ITBS patients would not undergo MR scanning. Secondly, since all the cases were outpatients, we could only screen suspected cases through MR images first and then further confirm these cases' final clinical diagnosis. All the included cases in this study presented typical fluidlike signals between the ITB and the lateral femoral condyle, so some chronic cases or cases with mild MRI signs were not included.

Our study has several limitations. First, this was a retrospective study. The control group was selected without considering the effect of the subject's physical activity level or the hip adductor function and myofascia, so there may have been a risk of selection bias in the control group. Clinical tests and measurements of the ITB tightness were not performed in either the ITBS or control groups, which might explain our results lack of mechanical support. Second, the selection of all subjects in the ITBS group was based on clinical diagnosis without histologic or pathologic 
evidence. The histological features of ITBS mainly include cyst, bursa, or synovial inflammation deep to the ITB $(4,15)$, but in our clinical practice, ITBS is almost always managed with conservative treatment; thus, it is difficult to obtain a pathological diagnosis. Third, the sample size of the ITBS group was relatively small. The relationship between each measurement parameter and ITBS was not very high, and further in-depth study would need an expanded sample size to establish a more robust correlation between these parameters and ITBS. Future research efforts should focus on the relationship between the patella and ITBS using a prospective longitudinal study and the relationship between ITBS and patellofemoral osteoarthritis.

\section{Conclusions}

ITBS is the result of a series of complex mechanisms. This study identified a high incidence of patellofemoral malalignment, particularly patella alta and lateral tilt, and a reduced distance between the lateral femoral epicondyle and the ITB in ITBS patients. We confirmed that a higher position or lateral tilt of the patella and a steeper anterior part of the lateral femoral condyle were associated with the development of ITBS, which is helpful in understanding and further exploring the mechanism of ITBS.

\section{Acknowledgments}

Funding: None.

\section{Footnote}

Conflicts of Interest: All authors have completed the ICMJE uniform disclosure form (available at http://dx.doi. org/10.21037/qims-20-1101). The authors have no conflicts of interest to declare.

Ethical Statement: This study was approved by the First Affiliated Hospital of Chongqing Medical University institutional review board (No. 2019-309), and the study protocol was in accordance with the Declaration of Helsinki (as revised in 2013). Individual consent for this retrospective analysis was waived.

Open Access Statement: This is an Open Access article distributed in accordance with the Creative Commons Attribution-NonCommercial-NoDerivs 4.0 International License (CC BY-NC-ND 4.0), which permits the non- commercial replication and distribution of the article with the strict proviso that no changes or edits are made and the original work is properly cited (including links to both the formal publication through the relevant DOI and the license). See: https://creativecommons.org/licenses/by-nc-nd/4.0/.

\section{References}

1. Taunton JE, Ryan MB, Clement DB, McKenzie DC, Lloyd-Smith DR, Zumbo BD. A retrospective casecontrol analysis of 2002 running injuries. Br J Sports Med 2002;36:95-101.

2. Pegrum J, Self A, Hall N. Iliotibial band syndrome. BMJ 2019;364:1980.

3. van Gent RN, Siem D, van Middelkoop M, van Os AG, Bierma-Zeinstra SMA, Koes BW. Incidence and determinants of lower extremity running injuries in long distance runners: a systematic review. Br J Sports Med 2007;41:469-80.

4. Strauss EJ, Kim S, Calcei JG, Park D. Iliotibial band syndrome: evaluation and management. J Am Acad Orthop Surg 2011;19:728-736.

5. Gunter P, Schwellnus MP. Local corticosteroid injection in iliotibial band friction syndrome in runners: a randomised controlled trial. Br J Sports Med 2004;38:269-72.

6. van der Worp MP, van der Horst N, de Wijer A, Backx FJ, Nijhuis-van der Sanden MW. Iliotibial band syndrome in runners: a systematic review. Sports Med 2012;42:969-92.

7. Baker RL, Souza RB, Fredericson M. Iliotibial band syndrome:Soft tissue and biomechanical factors in evaluation and treatment. PM R 2011;3:550-61.

8. Kim DY, Miyakawa S, Fukuda T, Takemura M. Sex differences in iliotibial band strain under different knee alignments. PM R 2020;12:479-85.

9. Noehren B, Schmitz A, Hempel R, Westlake C, Black W. Assessment of strength, flexibility, and running mechanics in men with iliotibial band syndrome. J Orthop Sports Phys Ther 2014;44:217-22.

10. Grau S, Krauss I, Maiwald C, Axmann D, Horstmann T, Best R. Kinematic classification of iliotibial band syndrome in runners. Scand J Med Sci Sports 2011;21:184-9.

11. Shen P, Mao D, Zhang C, Sun W, Song Q. Effects of running biomechanics on the occurrence of iliotibial band syndrome in male runners during an eight-week running programme-a prospective study. Sports Biomech 2019. doi: 10.1080/14763141.2019.1584235. [Epub ahead of 
print].

12. Everhart JS, Kirven JC, Higgins J, Hair A, Chaudhari AAMW, Flanigan DC. The relationship between lateral epicondyle morphology and iliotibial band friction syndrome: a matched case-control study. Knee 2019;26:1198-203.

13. Vieira EL, Vieira EA, da Silva RT, Berlfein PA, Abdalla RJ, Cohen M. An anatomic study of the iliotibial tract. Arthroscopy 2007;23:269-74.

14. Birnbaum K, Siebert CH, Pandorf T, Schopphoff E, Prescher A, Niethard FU. Anatomical and biomechanical investigations of the iliotibial tract. Surg Radiol Anat 2004;26:433-46.

15. Flato R, Passanante GJ, Skalski MR, Patel DB, White EA, Matcuk GR. The iliotibial tract: Imaging, anatomy, injuries, and other pathology. Skeletal Radiol 2017;46:605-22.

16. Merican AM, Iranpour F, Amis AA. Iliotibial band tension reduces patellar lateral stability. J Orthop Res 2009;27:335-9.

17. Muhle C, Ahn JM, Yeh L, Bergman GA, Boutin RD, Schweitzer M, Jacobson JA, Haghighi P, Trudell DJ, Resnick D. Iliotibial band friction syndrome: MR imaging findings in 16 patients and MR arthrographic study of six cadaveric knees. Radiology 1999;212:103-10.

18. Ekman EF, Pope T, Martin DF, Curl WW. Magnetic resonance imaging of iliotibial band syndrome. Am J Sports Med 1994;22:851-4.

19. Insall J, Salvati E. Patella position in the normal knee joint. Radiology 1971;101:101-4.

20. Wittstein JR, Bartlett EC, Easterbrook J, Byrd JC. Magnetic resonance imaging evaluation of patellofemoral malalignment. Arthroscopy 2006;22:643-9.

21. Matcuk GR Jr, Cen SY, Keyfes V, Patel DB, Gottsegen CJ, White EA. Superolateral hoffa fat-pad edema and patellofemoral maltracking: Predictive modeling. AJR Am J Roentgenol 2014;203:W207-12.

22. Fairclough J, Hayashi K, Toumi H, Lyons K, Bydder G, Phillips N, Best TM, Benjamin M. The functional anatomy of the iliotibial band during flexion and extension of the knee: Implications for understanding iliotibial band syndrome. J Anat 2006;208:309-16.

23. Ward SR, Terk MR, Powers CM. Patella alta: association with patellofemoral alignment and changes in contact area during weight-bearing. J Bone Joint Surg Am 2007;89:1749-55.

24. Munch JL, Sullivan JP, Nguyen JT, Mintz D, Green DW, Shubin Stein BE, Strickland S. Patellar articular overlap on MRI is a simple alternative to conventional measurements of patellar height. Orthop J Sports Med 2016;4:2325967116656328.

25. O'Keeffe SA, Hogan BA, Eustace SJ, Kavanagh EC. Overuse injuries of the knee. Magn Reson Imaging Clin N Am 2009;17:725-39.

26. Kim JH, Lee SK. Superolateral hoffa fat pad edema and patellofemoral maltracking: Systematic review and MetaAnalysis. AJR Am J Roentgenol 2020;215:545-58.

27. Widjajahakim R, Roux M, Jarraya M, Roemer FW, Neogi T, Lynch JA, Lewis CE, Torner JC, Felson DT, Guermazi A, Stefanik JJ. Relationship of Trochlear Morphology and Patellofemoral Joint alignment to superolateral hoffa Fat Pad edema on MR images in individuals with or at risk for Osteoarthritis of the Knee: the MOST Study. Radiology 2017;284:806-14.

28. Subhawong TK, Eng J, Carrino JA, Chhabra A. Superolateral Hoffa's fat pad edema: association with patellofemoral maltracking and impingement. AJR Am J Roentgenol 2010;195:1367-73.

29. Li J, Sheng B, Yu F, Guo C, Lv F, Lv F, Yang H. Quantitative magnetic resonance imaging in patellar tendon-lateral femoral condyle friction syndrome: relationship with subtle patellofemoral instability. Skeletal Radiol 2019;48:1251-9.

30. Grantham WJ, Aman ZS, Brady AW, Rosenberg SI, Turnbull TL, Storaci HW, Dornan GJ, LaPrade RF. Medial Patellotibial Ligament Reconstruction Improves Patella Tracking when Combined with Medial Patellofemoral Reconstruction: an In Vitro Kinematic Study. Arthroscopy2020;36:2501-9.

31. Otsuki S, Nakajima M, Okamoto Y, Oda S, Hoshiyama Y, Iida G, Neo M. Correlation between varus knee malalignment and patellofemoral osteoarthritis. Knee Surg Sports Traumatol Arthrosc 2016;24:176-81.

32. Tsavalas N, Katonis P, Karantanas AH. Knee joint anterior malalignment and patellofemoral osteoarthritis: an MRI study. Eur Radiol2012;22:418-28.

33. Haj-Mirzaian A, Guermazi A, Pishgar F, Pourvaziri A, Roemer FW, Sereni C, Hakky M, Zikria B, Stefanik JJ, Demehri S. Association of Patella Alta with Worsening of Patellofemoral Osteoarthritis-related Structural Damage: Data from the Osteoarthritis Initiative. Osteoarthritis Cartilage 2019;27:278-85.

34. Ambra LF, Hinckel BB, Arendt EA, Farr J, Gomoll AH. Anatomic risk factors for focal cartilage lesions in the patella and trochlea: a Case-Control study. Am J Sports Med 2019;47:2444-53. 
35. van der Heijden RA, de Vries BA, Poot DHJ, van Middelkoop M, Bierma-Zeinstra SMA, Krestin GP, Oei EHG. Quantitative volume and dynamic contrast- enhanced MRI derived perfusion of the infrapatellar fat pad in patellofemoral pain. Quant Imaging Med Surg 2021;11:133-42.

Cite this article as: Li J, Sheng B, Qiu L, Yu F, Lv FJ, Lv FR, Yang H. A quantitative MRI investigation of the association between iliotibial band syndrome and patellofemoral malalignment. Quant Imaging Med Surg 2021;11(7):32093218. doi: 10.21037/qims-20-1101 